\title{
The relevance of the Laplacian of intracule and extracule density distributions for analyzing electron-electron interactions in molecules
}

\author{
Xavier Fradera, Miquel Duran, ${ }^{\text {a) }}$ and Jordi Mestres ${ }^{\text {a) }}$ \\ Department of Chemistry and Institute for Computational Chemistry, University of Girona, 17071 Girona, \\ Catalonia, Spain
}

(Received 24 April 1997; accepted 28 May 1997)

\begin{abstract}
A topological analysis of intracule and extracule densities and their Laplacians computed within the Hartree-Fock approximation is presented. The analysis of the density distributions reveals that among all possible electron-electron interactions in atoms and between atoms in molecules only very few are located rigorously as local maxima. In contrast, they are clearly identified as local minima in the topology of Laplacian maps. The conceptually different interpretation of intracule and extracule maps is also discussed in detail. An application example to the $\mathrm{C}_{2} \mathrm{H}_{2}, \mathrm{C}_{2} \mathrm{H}_{4}$, and $\mathrm{C}_{2} \mathrm{H}_{6}$ series of molecules is presented. (C) 1997 American Institute of Physics. [S0021-9606(97)00833-7]
\end{abstract}

\section{INTRODUCTION}

In recent years, the study of the electronic characteristics of atoms and molecules has experienced a novel impulse. The constant urge to explore new alternative ways for analyzing the information contained in the electronic wave function of molecular systems has driven the attention from the widely established topological analyses based on oneelectron densities ${ }^{1}$ to those based on electron-pair densities. ${ }^{2}$ However, an electron-pair density, ${ }^{3} \Gamma\left(\mathbf{r}_{1}, \mathbf{r}_{2}\right)$, is a function of six variables and hence its topology is difficult to represent and analyze in detail. To overcome this inconvenience, one can make use of the electron intracule and extracule densities, ${ }^{4}$ which have the advantage of reducing the dimensionality of the electron-pair density without losing its original two-electron character.

For a pair of electrons, definition of the intracule coordinates as $\mathbf{r}=\mathbf{r}_{1}-\mathbf{r}_{2}$ and the extracule coordinates as $\mathbf{R}=\left(\mathbf{r}_{1}+\mathbf{r}_{2}\right) / 2$ allows us to express the intracule, $I(\mathbf{r})$, and the extracule, $E(\mathbf{R})$, densities as

$$
\begin{aligned}
& I(\mathbf{r})=\int \Gamma\left(\mathbf{r}_{1}, \mathbf{r}_{2}\right) \delta\left[\left(\mathbf{r}_{1}-\mathbf{r}_{2}\right)-\mathbf{r}\right] d \mathbf{r}_{1} d \mathbf{r}_{2}, \\
& E(\mathbf{R})=\int \Gamma\left(\mathbf{r}_{1}, \mathbf{r}_{2}\right) \delta\left[\left(\mathbf{r}_{1}+\mathbf{r}_{2}\right) / 2-\mathbf{R}\right] d \mathbf{r}_{1} d \mathbf{r}_{2} .
\end{aligned}
$$

Accordingly, $I(\mathbf{r})$ and $E(\mathbf{R})$ are the probability density functions for the interparticle distance and for the center of mass of an electron pair, respectively. $I(\mathbf{r})$ is invariant to translations of the molecule and has a center of inversion at the origin. In contrast, $E(\mathbf{R})$ reflects the spatial arrangement of the nuclear framework and its origin depends upon the molecular coordinates.

Besides $I(\mathbf{r})$ and $E(\mathbf{R})$, evaluation of their respective Laplacians $\left[\nabla^{2} I(\mathbf{r})\right.$ and $\left.\nabla^{2} E(\mathbf{R})\right]$ has been barely considered. It is well-known that the sign of the Laplacian of a function allows us to detect where the function is locally concentrated (negative values) and where it is locally depleted (positive values). For instance, this property gives to

\footnotetext{
a) Authors to whom correspondence should be addressed.
}

the Laplacian of the one-electron density the ability to reveal the shell structure of atoms in molecules. ${ }^{1}$ Extension of its use to molecular electron-pair density distributions is one of the goals of the present work.

In addition, the present knowledge of the topological properties of $I(\mathbf{r})$ and $E(\mathbf{R})$ is limited and so that of their Laplacian distributions too. In this sense, a well established property of $I(\mathbf{r})$ is the existence of the electron coalescence cusp at $\mathbf{r}=0 . .^{5-8}$ However, approximate $I(\mathbf{r})$ from HartreeFock (HF) wave functions do not possess this electron coalescence cusp, as their spherically averaged gradient vanishes at the origin. Despite this peculiar behavior, it has been recently shown that the main topological features of accurate $I(\mathbf{r})$ are already manifested in approximate $I(\mathbf{r})$ from $\mathrm{HF}$ calculations. ${ }^{2}$ Thus, as a first approximation, in this work $I(\mathbf{r})$ and $E(\mathbf{R})$ densities and their corresponding Laplacians will be computed at the HF level of theory.

So far, $I(\mathbf{r})$ and $E(\mathbf{R})$ calculations have been mainly performed on atomic systems ${ }^{8-10}$ and small molecules. ${ }^{1-13}$ Calculations of their Laplacians are even more uncommon and have been restricted to atoms. ${ }^{9,10}$ In this aspect, the recent description of a more efficient algorithm to compute $I(\mathbf{r})$ and $E(\mathbf{R})$ (Ref. 14) provides a way to scrutinize in more detail the topology of electron-pair densities in small molecules and to extend this kind of analyses to larger molecules. $^{2}$

The aim of this work is to study the practically unexplored topology of $\nabla^{2} I(\mathbf{r})$ and $\nabla^{2} E(\mathbf{R})$ distributions in molecules and to show their relevance for identifying electronelectron interactions as compared to $I(\mathbf{r})$ and $E(\mathbf{R})$ distributions. The following sections contain, first, a description of the computational methods used and, second, an application example to the $\mathrm{C}_{2} \mathrm{H}_{2}, \mathrm{C}_{2} \mathrm{H}_{4}$, and $\mathrm{C}_{2} \mathrm{H}_{6}$ series of molecules.

\section{COMPUTATIONAL METHODS}

Molecular geometries were optimized at the $\mathrm{HF} / 6-31 \mathrm{G}^{*}$ level of theory by means of the GAUSSIAN-94 series of programs. ${ }^{15}$ The resulting molecular coordinates were then mass-centered to make the origin of $E(\mathbf{R})$ and 


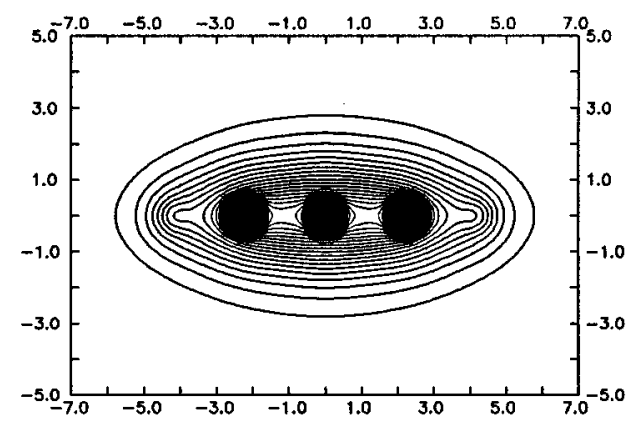

(a)

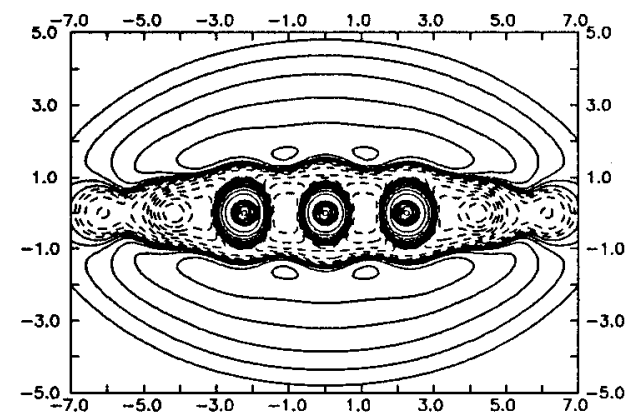

(c)

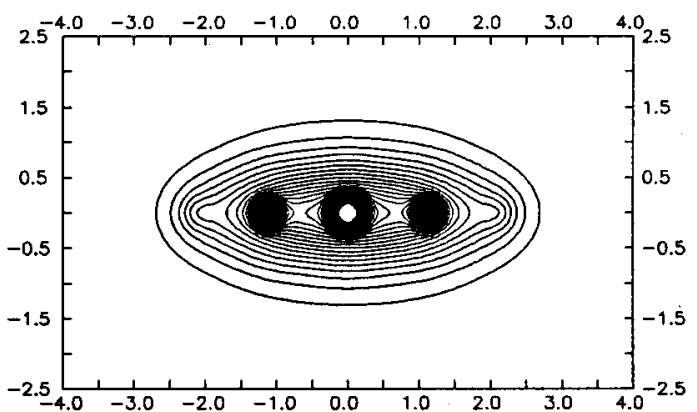

(b)

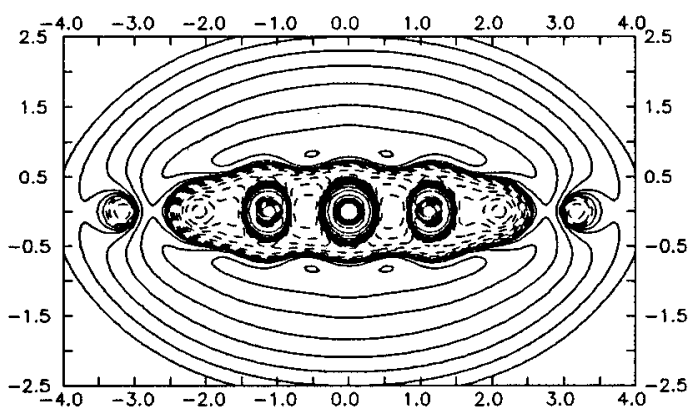

(d)

FIG. 1. Topological maps for the $\mathrm{C}_{2} \mathrm{H}_{2}$ molecule: (a) intracule density (in contours of 0.1 a.u.), (b) extracule density (in contours of 1.0 a.u.), (c) Laplacian of the intracule density (in contours of $\pm 0.01 \times 2^{n}$ a.u., $n=0,1,2, \ldots$ ), and (d) Laplacian of the extracule density (in contours of $\pm 0.1 \times 2^{n}$ a.u., $n=0,1,2, \ldots)$. Positive values are depicted in solid lines and negative values in dashed lines.

$\nabla^{2} E(\mathbf{R})$ distributions correspond to the molecular center of mass. Calculations of $I(\mathbf{r})$ and $E(\mathbf{R})$ densities and their respective Laplacian distributions were performed following the algorithm recently described by Cioslowski and Liu, ${ }^{14}$ which allows for fast evaluations on large grids of points. In all calculations, the integral neglect threshold was set to $10^{-5} \cdot{ }^{14}$ Characterization of electron-electron interactions was carried out through location of local maxima in electronpair density maps and local minima in their Laplacian distributions.

\section{APPLICATION EXAMPLES}

The $\mathrm{C}_{2} \mathrm{H}_{2}$ and $\mathrm{C}_{2} \mathrm{H}_{4}$ molecules have been selected as application examples. The simplicity conferred by their high symmetry makes them particularly adequate to analyze visually the complex topology of the different electron-pair and Laplacian distributions and to perform a detailed study of the variety of electron-electron interactions present in these molecules. For the sake of completeness, the $\mathrm{C}_{2} \mathrm{H}_{6}$ molecule will be later considered in the Discussion to examine the trends followed by those distributions in this series of molecules. For all molecules, the two carbon atoms define the $x$ axis, while positions of the hydrogens define the $x z$ plane in $\mathrm{C}_{2} \mathrm{H}_{4}$.

\section{A. $\mathrm{C}_{2} \mathrm{H}_{2}$}

Figure 1 depicts the set of $I(\mathbf{r}), E(\mathbf{R}), \nabla^{2} I(\mathbf{r})$, and $\nabla^{2} E(\mathbf{R})$ topological maps for $\mathrm{C}_{2} \mathrm{H}_{2}$. At first glance, the topologies of $I(\mathbf{r})$ and $E(\mathbf{R})$ [Figs. 1(a) and 1(b)], on one side, and $\nabla^{2} I(\mathbf{r})$ and $\nabla^{2} E(\mathbf{R})$ [Figs. 1(c) and 1(d)], on the other side, look qualitatively very similar. However, this visual impression is only caused by the high symmetry of the $\mathrm{C}_{2} \mathrm{H}_{2}$ molecule. As will be shown below, the interpretation of local maxima in $I(\mathbf{r})$ and $E(\mathbf{R})$ and local minima in $\nabla^{2} I(\mathbf{r})$ and $\nabla^{2} E(\mathbf{R})$ is conceptually different in intracule and extracule distributions, despite being visually similar in this case.

From a qualitative point of view, another visual aspect worth being remarked from Figs. 1(c) and 1(d) is that the topology of the molecular $\nabla^{2} I(\mathbf{r})$ and $\nabla^{2} E(\mathbf{R})$ maps reveals the shell structure of atoms in the molecule, as previously noted by Sarasola et al. ${ }^{10}$ for isolated atoms. In particular, the two shells of carbon atoms can be clearly identified. However, it must be clarified that, although visually similar to the shell structure revealed by the Laplacian of oneelectron densities, the interpretation of shells in the topology of $\nabla^{2} I(\mathbf{r})$ and $\nabla^{2} E(\mathbf{R})$ distributions (in terms of the type and number of electrons contributing to these shells) is essentially different. For instance, one must be aware that carbon core-electron pairs will all furnish the carbon inner-shell but 
TABLE I. Internuclear distances $\left(\left|\mathbf{D}_{A B}\right|\right.$ in a.u.) and coordinates ( $x$ in a.u.), intracule and extracule densities $[I(\mathbf{r})$ and $E(\mathbf{R})$ in a.u. $]$ and Laplacian values $\left[\nabla^{2} I(\mathbf{r})\right.$ and $\nabla^{2} E(\mathbf{R})$ in a.u. $]$ of the different electron-electron interactions assigned to local minima in Laplacian maps for $\mathrm{C}_{2} \mathrm{H}_{2}$.

\begin{tabular}{|c|c|c|c|c|c|c|c|}
\hline \multirow[b]{2}{*}{ Interaction } & \multirow[b]{2}{*}{$\left|\mathbf{D}_{A B}\right|$} & \multicolumn{3}{|c|}{ Intracule } & \multicolumn{3}{|c|}{ Extracule } \\
\hline & & $x$ & $I(\mathbf{r})$ & $\nabla^{2} I(\mathbf{r})$ & $x$ & $E(\mathbf{R})$ & $\nabla^{2} E(\mathbf{R})$ \\
\hline 0 & $\cdots$ & 0.000 & 15.828 & -2042.8 & 0.000 & 252.773 & -132523.0 \\
\hline$\left\{\mathrm{C}_{i i}\right\}$ & 0.000 & $\ldots$ & $\ldots$ & $\ldots$ & 1.120 & 70.848 & -33244.8 \\
\hline$\left\{\mathrm{C}_{i} \mathrm{C}_{j}\right\}$ & 2.240 & 2.240 & 16.354 & -2075.2 & $\ldots$ & $\ldots$ & $\ldots$ \\
\hline$\left\{\mathrm{C}_{i} \mathrm{H}_{i}\right\}$ & 1.997 & $\ldots$ & $\ldots$ & $\ldots$ & 2.111 & 6.204 & -359.511 \\
\hline$\left\{\mathrm{C}_{i} \mathrm{H}_{j}\right\}$ & 4.237 & 4.220 & 0.808 & -11.340 & $\ldots$ & $\ldots$ & $\ldots$ \\
\hline$\left\{\mathrm{H}_{i i}\right\}$ & 0.000 & $\ldots$ & $\ldots$ & $\ldots$ & 3.112 & 0.298 & -0.789 \\
\hline$\left\{\mathrm{H}_{i} \mathrm{H}_{j}\right\}$ & 6.235 & 6.054 & 0.075 & -0.099 & $\ldots$ & $\ldots$ & $\cdots$ \\
\hline
\end{tabular}

that, in addition, contributions from the valence-electron pairs having a given probability of either being at short interelectron distances [in $\nabla^{2} I(\mathbf{r})$ ] or having their centers of mass in the neighborhood of the carbon center $\left[\right.$ in $\left.\nabla^{2} E(\mathbf{R})\right]$ should be also considered. A more detailed quantitative discussion on this specific subject will be found elsewhere. ${ }^{16}$

Table I collects a list of some of the local minima located in the topology of the $\nabla^{2} I(\mathbf{r})$ and $\nabla^{2} E(\mathbf{R})$ maps depicted in Fig. 1. Each local minimum is characterized by its position along the $x$ axis and the values of the corresponding density and Laplacian on top of this position. Due to the above mentioned inherent symmetry of these maps, all unique local minima in Laplacian maps (and all unique local maxima in density maps) can be located by considering only one half side of the maps in Fig. 1 (left or right). Thus, for the sake of simplicity, only coordinates of the local minima in $\nabla^{2} I(\mathbf{r})$ [Fig. 1(c)] and $\nabla^{2} E(\mathbf{R})$ [Fig. 1(d)] located in the right-hand side of the maps are presented.

In Table I, each local minimum has been mainly associated with a set of electron-electron interactions, ${ }^{17}$ which have been labelled following a particular notation: $\left\{\mathrm{C}_{i} \mathrm{C}_{j}\right\}$ refers to the set of intercarbon electron interactions; $\left\{\mathrm{C}_{i} \mathrm{H}_{j}\right\}$ to interatomic electron interactions between a carbon and its bonded hydrogen; $\left\{\mathrm{C}_{i} \mathrm{H}_{j}\right\}$ to interatomic electron interactions between a carbon and a hydrogen bonded to the other carbon atom; $\left\{\mathrm{H}_{i} \mathrm{H}_{j}\right\}$ to interhydrogen interactions; and $\left\{\mathrm{C}_{i i}\right\}$ and $\left\{\mathrm{H}_{i i}\right\}$ to intra-atomic interactions. The interaction labeled as 0 refers to the local minimum at the origin of the Laplacian maps (local maximum in density maps). Actually, it is a very special point in all electron-pair distributions as it gathers usually contributions from different electron-electron interactions. For instance, at the HF level of theory, all atoms collectively contribute to the origin in intracule distributions whereas, if molecular coordinates are previously masscentered, all electron pairs being invariable upon a symmetry-inversion operation will contribute to the origin in extracule distributions.

The first interesting aspect that can be extracted out of Table I is that, as regards to the assignment of the 6 types of electron-electron interactions to local minima in Laplacian distributions, $\nabla^{2} I(\mathbf{r})$ and $\nabla^{2} E(\mathbf{R})$ fully complement each other. Therefore, while the sets of $\left\{\mathrm{C}_{i} \mathrm{C}_{j}\right\},\left\{\mathrm{C}_{i} \mathrm{H}_{j}\right\}$, and
$\left\{\mathrm{H}_{i} \mathrm{H}_{j}\right\}$ interactions can be located in $\nabla^{2} I(\mathbf{r})$, interactions corresponding to $\left\{\mathrm{C}_{i i}\right\},\left\{\mathrm{C}_{i} \mathrm{H}_{j}\right\}$, and $\left\{\mathrm{H}_{i i}\right\}$ can be identified in $\nabla^{2} E(\mathbf{R})$. In $\nabla^{2} I(\mathbf{r})$, the $\left\{\mathrm{C}_{i i}\right\}$ and $\left\{\mathrm{H}_{i i}\right\}$ interactions contribute to the origin, whereas $\left\{\mathrm{C}_{i} \mathrm{H}_{i}\right\}$ interactions are not separately appreciated because they are contained into the well of the stronger $\left\{\mathrm{C}_{i} \mathrm{C}_{j}\right\}$ interactions. In $\nabla^{2} E(\mathbf{R})$, the interactions $\left\{\mathrm{C}_{i} \mathrm{C}_{j}\right\}$ and $\left\{\mathrm{H}_{i} \mathrm{H}_{j}\right\}$ are invariable upon a symmetry-inversion operation and, thus, they contribute to the origin, while in this case the set of $\left\{\mathrm{C}_{i} \mathrm{H}_{j}\right\}$ interactions is masked in the domains of the deep well of the $\left\{\mathrm{C}_{i i}\right\}$ interactions.

Less precise assignments can be performed when attempting this kind of analysis in $I(\mathbf{r})$ and $E(\mathbf{R})$ maps, where only two local maxima are rigourously characterized. The local maximum at the origin is furnished by $\left\{\mathrm{C}_{i i}\right\}$ and $\left\{\mathrm{H}_{i i}\right\}$ interactions in $I(\mathbf{r})$ and $\left\{\mathrm{C}_{i} \mathrm{C}_{j}\right\}$ and $\left\{\mathrm{H}_{i} \mathrm{H}_{j}\right\}$ interactions in $E(\mathbf{R})$. The other local maximum contains the interactions $\left\{\mathrm{C}_{i} \mathrm{H}_{i}\right\}$ and $\left\{\mathrm{C}_{i} \mathrm{C}_{j}\right\}$ in $\mathrm{I}(\mathbf{r})$ and the interactions $\left\{\mathrm{C}_{i} \mathrm{H}_{j}\right\}$ and $\left\{\mathrm{C}_{i i}\right\}$ in $E(\mathbf{R})$. The sets of $\left\{\mathrm{C}_{i} \mathrm{H}_{j}\right\}$ and $\left\{\mathrm{C}_{i} \mathrm{H}_{i}\right\}$ interactions can be recognized only as shoulders in the topology of $I(\mathbf{r})$ and $E(\mathbf{R})$ maps, respectively.

In summary, for the sake of clarity and considering only the right-hand side of $\nabla^{2} I(\mathbf{r})$ and $\nabla^{2} E(\mathbf{R})$ maps depicted in Figs. 1(c) and 1(d), respectively, the assignment and relative spatial arrangement of the different types of electronelectron interactions present in $\mathrm{C}_{2} \mathrm{H}_{2}$ can be illustrated schematically as

$$
\begin{aligned}
& \nabla^{2} I(\mathbf{r}): \quad\left\{\mathrm{C}_{i i}\right\},\left\{\mathrm{H}_{i i}\right\} \quad\left\{\mathrm{C}_{i} \mathrm{H}_{i}\right\},\left\{\mathrm{C}_{i} \mathrm{C}_{j}\right\} \quad\left\{\mathrm{C}_{i} \mathrm{H}_{j}\right\} \quad\left\{\mathrm{H}_{i} \mathrm{H}_{j}\right\} \\
& \nabla^{2} E(\mathbf{R}): \quad\left\{\mathrm{C}_{i} \mathrm{C}_{j}\right\},\left\{\mathrm{H}_{i} \mathrm{H}_{j}\right\} \quad\left\{\mathrm{C}_{i} \mathrm{H}_{j}\right\},\left\{\mathrm{C}_{i i}\right\} \quad\left\{\mathrm{C}_{i} \mathrm{H}_{i}\right\} \quad\left\{\mathrm{H}_{i i}\right\}
\end{aligned}
$$

which may help to appreciate the conceptual difference between $\nabla^{2} I(\mathbf{r})$ and $\nabla^{2} E(\mathbf{R})$.

\section{B. $\mathrm{C}_{2} \mathrm{H}_{4}$}

The series of $I(\mathbf{r}), E(\mathbf{R}), \nabla^{2} I(\mathbf{r})$, and $\nabla^{2} E(\mathbf{R})$ topological maps corresponding to the $\mathrm{C}_{2} \mathrm{H}_{4}$ molecule, in the plane containing all atoms, is depicted in Fig. 2. As in the case of the $\mathrm{C}_{2} \mathrm{H}_{2}$ molecule, the symmetry of $\mathrm{C}_{2} \mathrm{H}_{4}$ confers to $I(\mathbf{r})$ and $E(\mathbf{R})$ [Figs. 2(a) and 2(b)], on one side, and to $\nabla^{2} I(\mathbf{r})$ and $\nabla^{2} E(\mathbf{R})$ [Figs. 2(c) and 2(d)], on the other side, a qualitatively similar look. However, as will be shown be- 


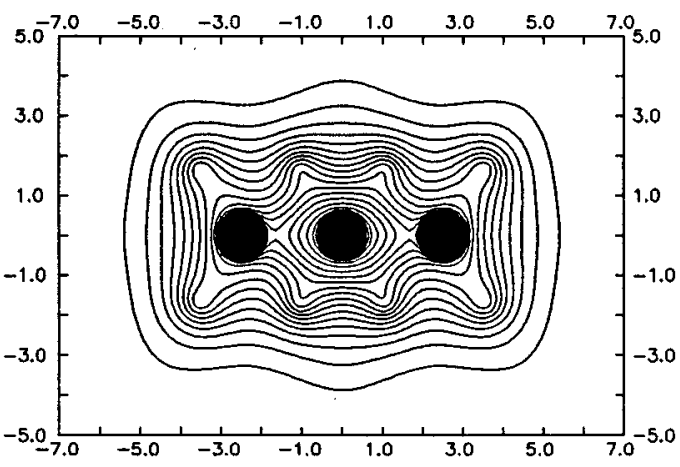

(a)

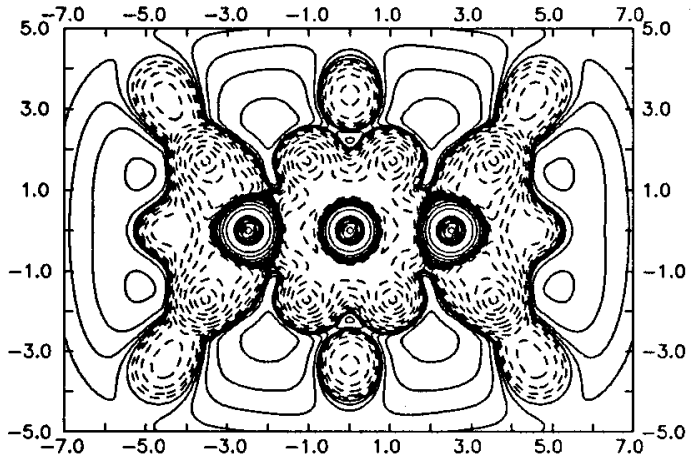

(c)

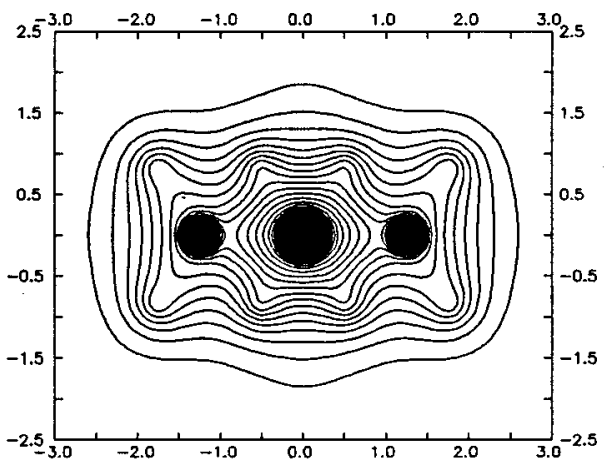

(b)

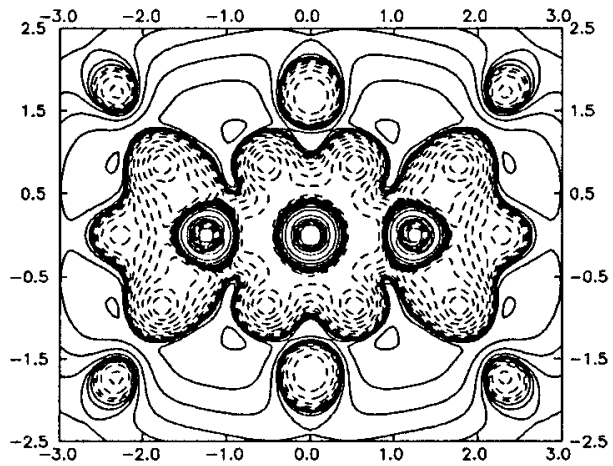

(d)

FIG. 2. Topological maps for the $\mathrm{C}_{2} \mathrm{H}_{4}$ molecule: (a) intracule density (in contours of 0.1 a.u.), (b) extracule density (in contours of 1.0 a.u.), (c) Laplacian of the intracule density (in contours of $\pm 0.01 \times 2^{n}$ a.u., $n=0,1,2, \ldots$ ), and (d) Laplacian of the extracule density (in contours of $\pm 0.1 \times 2^{n}$ a.u., $n=0,1,2, \ldots)$. Positive values are depicted in solid lines and negative values in dashed lines.

low, their respective interpretation is essentially different. Note also that, as commented above, the shell structure of atoms in the molecule is revealed by the $\nabla^{2} I(\mathbf{r})$ [Fig. 2(c)] and $\nabla^{2} E(\mathbf{R})$ [Fig. 2(d)] distributions.

Table II gathers some of the local minima located in the topology of the $\nabla^{2} I(\mathbf{r})$ and $\nabla^{2} E(\mathbf{R})$ maps depicted in Fig. 2, characterized by their position on the $x z$ plane and the values of the corresponding density and Laplacian on top of this position. In this case, due to the symmetry of $\mathrm{C}_{2} \mathrm{H}_{4}$, consid- eration of only one quarter of the maps in Fig. 2 is sufficient to locate the set of unique local maxima and minima in density and Laplacian maps, respectively, and thus only coordinates of the local minima in $\nabla^{2} I(\mathbf{r})$ [Fig. 2(c)] and $\nabla^{2} E(\mathbf{R})$ [Fig. 2(d)] located in the upper-right quarter of the maps are presented.

In Table II, each local minimum has been mainly associated with a set of electron-electron interactions. In comparison to the electron-electron interactions discussed above

TABLE II. Internuclear distances $\left(\left|\mathbf{D}_{A B}\right|\right.$ in a.u.) and interelectron distances $\left(\left|\mathbf{r}_{a b}\right|\right.$ in a.u.), interelectron centers of mass $\left(\left|\mathbf{R}_{a b}\right|\right.$ in a.u.), coordinates $(x z$ in a.u.), intracule and extracule densities $[I(\mathbf{r})$ and $E(\mathbf{R})$ in a.u. $]$ and Laplacian values $\left[\nabla^{2} I(\mathbf{r})\right.$ and $\nabla^{2} E(\mathbf{R})$ in a.u. of the different electron-electron interactions assigned to local minima in Laplacian maps for $\mathrm{C}_{2} \mathrm{H}_{4}$.

\begin{tabular}{|c|c|c|c|c|c|c|c|c|c|}
\hline \multirow[b]{2}{*}{ Interaction } & \multirow[b]{2}{*}{$\left|\mathbf{D}_{\mathrm{AB}}\right|$} & \multicolumn{4}{|c|}{ Intracule } & \multicolumn{4}{|c|}{ Extracule } \\
\hline & & $\left|\mathbf{r}_{\mathrm{ab}}\right|$ & $(x, z)$ & $I(\mathbf{r})$ & $\nabla^{2} I(\mathbf{r})$ & $\left|\mathbf{R}_{a b}\right|$ & $(x, z)$ & $E(\mathbf{R})$ & $\nabla^{2} E(\mathbf{R})$ \\
\hline 0 & $\ldots$ & 0.000 & $(0.000,0.000)$ & 15.847 & -2036.3 & 0.000 & $(0.000,0.000)$ & 253.160 & -132122.5 \\
\hline$\left\{\mathrm{C}_{i i}\right\}$ & 0.000 & $\ldots$ & $\ldots$ & $\ldots$ & $\ldots$ & 0.000 & $(1.244,0.000)$ & 66.931 & -32984.0 \\
\hline$\left\{\mathrm{C}_{i} \mathrm{C}_{j}\right\}$ & 2.489 & 2.489 & $(2.489,0.000)$ & 15.869 & -2063.9 & $\ldots$ & $\ldots$ & $\ldots$ & $\ldots$ \\
\hline$\left\{\mathrm{C}_{i} \mathrm{H}_{i}\right\}$ & 2.033 & 2.018 & $(1.063,1.715)$ & 1.047 & -11.707 & 1.009 & $(1.776,0.857)$ & 6.619 & -382.565 \\
\hline$\left\{\mathrm{C}_{i} \mathrm{H}_{j}\right\}$ & 3.958 & 3.945 & $(3.553,1.715)$ & 0.858 & -12.042 & 1.972 & $(0.532,0.857)$ & 8.672 & -374.297 \\
\hline$\left\{\mathrm{H}_{i i}\right\}$ & 0.000 & $\ldots$ & $\ldots$ & $\ldots$ & $\ldots$ & 0.000 & $(2.319,1.727)$ & 0.350 & -0.957 \\
\hline$\left\{\mathrm{H}_{i} \mathrm{H}_{i}\right\}$ & 3.456 & 3.369 & $(0.000,3.369)$ & 0.177 & -0.199 & $\ldots$ & $(2.292,0.000)$ & 2.051 & -4.132 \\
\hline$\left\{\mathrm{H}_{i} \mathrm{H}_{j}(c i s)\right\}$ & 4.633 & 4.579 & $(4.579,0.000)$ & 0.267 & -0.133 & $\cdots$ & $(0.000,1.692)$ & 1.410 & -6.224 \\
\hline$\left\{\mathrm{H}_{i} \mathrm{H}_{j}(\right.$ trans $\left.)\right\}$ & 5.780 & 5.653 & $(4.562,3.339)$ & 0.082 & -0.114 & $\cdots$ & $\ldots$ & $\ldots$ & $\cdots$ \\
\hline
\end{tabular}


for the $\mathrm{C}_{2} \mathrm{H}_{2}$ molecule, a new set of $\left\{\mathrm{H}_{i} \mathrm{H}_{i}\right\}$ interactions have to be taken into account to refer to interhydrogen electron interactions between hydrogens linked to the same carbon. Furthermore, $\left\{\mathrm{H}_{i} \mathrm{H}_{j}\right\}$ interactions are now separated into $\left\{\mathrm{H}_{i} \mathrm{H}_{j}(\right.$ cis $\left.)\right\}$ and $\left\{\mathrm{H}_{i} \mathrm{H}_{j}\right.$ (trans) $\}$ depending on the relative situation of the two hydrogens linked to different carbon atoms.

The assignment of the main types of electron-electron interactions in $\mathrm{C}_{2} \mathrm{H}_{4}$ to local minima in $\nabla^{2} I(\mathbf{r})$ and $\nabla^{2} E(\mathbf{R})$ maps is also is also included in Table II. As can be observed, $\nabla^{2} I(\mathbf{r})$ and $\nabla^{2} E(\mathbf{R})$ distributions still complement each other in the resolution of those interactions contributing to the respective origins; in $\nabla^{2} I(\mathbf{r})$, while the sets of $\left\{\mathrm{C}_{i i}\right\}$ and $\left\{\mathrm{H}_{i i}\right\}$ interactions collectively contribute to the local minima at the origin, they can be separately identified in the $\nabla^{2} E(\mathbf{R})$ map; in $\nabla^{2} E(\mathbf{R})$, the two sets of $\left\{\mathrm{C}_{i} \mathrm{C}_{j}\right\}$ and $\left\{\mathrm{H}_{i} \mathrm{H}_{j}\right.$ (trans) $\}$ interactions furnish the origin, but they can be clearly discriminated in the topology of the $\nabla^{2} I(\mathbf{r})$ map. For comparison, only two local maxima were identified in $I(\mathbf{r})$ [Fig. 2(a)] and $E(\mathbf{R})$ [Fig. 2(b)] maps (which can be assigned to the same type of electron-electron interactions commented above for $\mathrm{C}_{2} \mathrm{H}_{2}$ ), although the sets of $\left\{\mathrm{C}_{i} \mathrm{H}_{i}\right\}$ and $\left\{\mathrm{C}_{i} \mathrm{H}_{j}\right\}$ interactions can be visually detected as shoulders in those maps.

In summary, the assignment of the 8 main types of electron-electron interactions to regions of local minima and their relative spatial arrangement in the top-right quarter of $\nabla^{2} I(\mathbf{r})$ and $\nabla^{2} E(\mathbf{R})$ maps in Figs. 2(c) and 2(d), respectively, can be depicted schematically as

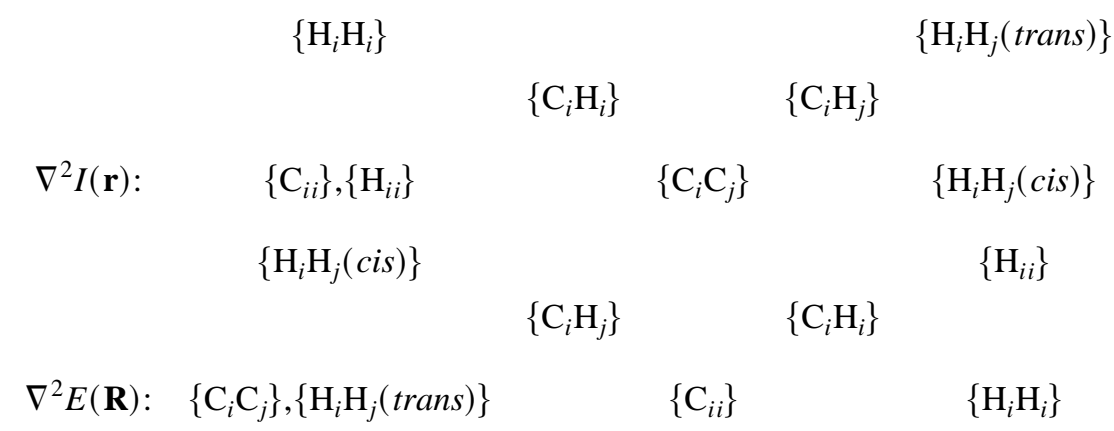

which again permits us to illustrate more clearly the subtle differences in the conceptual interpretation of $\nabla^{2} I(\mathbf{r})$ and $\nabla^{2} E(\mathbf{R})$ maps.

\section{Discussion}

Comparison of results presented above for the $\mathrm{C}_{2} \mathrm{H}_{2}$ and $\mathrm{C}_{2} \mathrm{H}_{4}$ molecules will allow explaining in more detail some aspects that, for the sake of clarity at that stage, were not rigorously discussed in the previous sections. In addition, throughout this section, reference to results obtained for the $\mathrm{C}_{2} \mathrm{H}_{6}$ molecule will be used, when necessary, to analyze the trends followed by this series of molecules.

A general observation from distance values in Tables I and II is that, except for the set of $\left\{\mathrm{C}_{i} \mathrm{C}_{j}\right\}$ interactions, electron-electron interatomic interactions are found at interelectron distances slightly closer than the corresponding internuclear distances. This trend is evident in electronelectron interactions between a carbon and a hydrogen atom, and is even more emphasized in those interactions between hydrogens. For instance, for $\mathrm{C}_{2} \mathrm{H}_{2}$ in Table I, interelectron $\left\{\mathrm{C}_{i} \mathrm{H}_{i}\right\}$ and $\left\{\mathrm{C}_{i} \mathrm{H}_{j}\right\}$ distances (1.982 and 4.220 a.u.) are 0.015 and 0.017 a.u. shorter than the corresponding internuclear $\mathrm{C}-\mathrm{H}$ distances (1.997 and 4.237 a.u.), respectively. In contrast, interelectron $\left\{\mathrm{H}_{i} \mathrm{H}_{j}\right\}$ distances (6.054 a.u.) appear 0.181 a.u. shorter than the $\mathrm{H}-\mathrm{H}$ distance (6.235 a.u.). A similar trend is found for the $\mathrm{C}_{2} \mathrm{H}_{4}$ molecule in Table II. In this case, interelectron $\left\{\mathrm{C}_{i} \mathrm{H}_{i}\right\}$ and $\left\{\mathrm{C}_{i} \mathrm{H}_{j}\right\}$ distances (2.018 and 3.945 a.u.) are 0.015 and 0.013 a.u. shorter than the corresponding internuclear $\mathrm{C}-\mathrm{H}$ distances (2.033 and 3.958 a.u.), while interelectron $\left\{\mathrm{H}_{i} \mathrm{H}_{i}\right\},\left\{\mathrm{H}_{i} \mathrm{H}_{j}(\right.$ cis $\left.)\right\}$, and $\left\{\mathrm{H}_{i} \mathrm{H}_{j}\right.$ (trans) $\}$ distances $(3.369,4.579$, and 5.653 a.u.) are, respectively, 0.087, 0.054, and 0.127 a.u. shorter than the corresponding $\mathrm{H}-\mathrm{H}$ internuclear distances (3.456, 4.633, and 5.780 a.u.). These results perfectly reflect the existence of chemical bonds and evidence the effects of electronic polarization between atoms in molecules, although they are expected to be exaggerated to some extent due to the use of Hartree-Fock wave functions in this work.

Furthermore, careful inspection of $I(\mathbf{r})$ and $E(\mathbf{R})$ values at the positions of the different local minima identified in $\nabla^{2} I(\mathbf{r})$ and $\nabla^{2} E(\mathbf{R})$ maps for $\mathrm{C}_{2} \mathrm{H}_{2}$ and $\mathrm{C}_{2} \mathrm{H}_{4}$ provides additional support to the assignments of electron-electron interactions qualitatively made above. As a general trend, $I(\mathbf{r})$ and $E(\mathbf{R})$ values in local minima assigned to comparable sets of interactions are smaller in $\mathrm{C}_{2} \mathrm{H}_{2}$ than in $\mathrm{C}_{2} \mathrm{H}_{4}$ (see Tables I and II). This trend is observed in those electronelectron interactions labeled as $\left\{\mathrm{C}_{i} \mathrm{H}_{i}\right\},\left\{\mathrm{C}_{i} \mathrm{H}_{j}\right\},\left\{\mathrm{H}_{i i}\right\}$, and $\left\{\mathrm{H}_{i} \mathrm{H}_{j}\right\}$. Therefore, for the set of $\left\{\mathrm{C}_{i} \mathrm{H}_{i}\right\}$ interactions in $\mathrm{C}_{2} \mathrm{H}_{2}$ and $\mathrm{C}_{2} \mathrm{H}_{4}, E(\mathbf{R})$ values of 6.204 and 6.619 a.u. are obtained; for the set of $\left\{\mathrm{C}_{i} \mathrm{H}_{j}\right\}$ interactions, $I(\mathbf{r})$ values of 0.808 and 0.858 a.u. are found; for $\left\{\mathrm{H}_{i i}\right\}$ interactions, $E(\mathbf{R})$ values of 0.298 and 0.350 a.u. are assigned; and for the set of $\left\{\mathrm{H}_{i} \mathrm{H}_{j}\right\}$ interactions, $I(\mathbf{r})$ values of $0.075\left(\right.$ in $\left.\mathrm{C}_{2} \mathrm{H}_{2}\right)$ and 0.082 and 
0.267 (for $\left\{\mathrm{H}_{i} \mathrm{H}_{j}(\right.$ cis $\left.)\right\}$ and $\left\{\mathrm{H}_{i} \mathrm{H}_{j}(\right.$ trans) $\}$ in $\mathrm{C}_{2} \mathrm{H}_{4}$ ) are encountered.

This same trend is also followed by $I(\mathbf{r})$ and $E(\mathbf{R})$ values at the origin (containing an ensemble of electronelectron interactions generally labeled as 0 in this work). For the particular case of the intracular coalescence density $[I(\mathbf{0})]$, a simple reason for this trend in the $\mathrm{C}_{2} \mathrm{H}_{2}, \mathrm{C}_{2} \mathrm{H}_{4}$, and $\mathrm{C}_{2} \mathrm{H}_{6}$ series of molecules can be found in the fact that, since under the Hartree-Fock approximation all atoms contribute to $I(\mathbf{0})$, the more hydrogens (i.e., the more electrons) the molecule possesses, the larger the $I(\mathbf{0})$ value. However, an alternative explanation from the perspective of one-electron densities can be derived when combining the findings reported in some recent studies. ${ }^{18,19}$ On the one hand, Ugalde and Sarasola ${ }^{18}$ showed that, under the Hartree-Fock approximation, evaluation of $I(\mathbf{0})$ can be performed through a functional of the one-electron density function as

$$
I(\mathbf{0})=1 / 4 \cdot\langle\rho\rangle ; \quad\langle\rho\rangle=\int \rho(\mathbf{r}) \rho(\mathbf{r}) d \mathbf{r} .
$$

On the other hand, and from a completely different point of view, Solà et $a l .{ }^{19}$ reported $\langle\rho\rangle$ values for atoms from $\mathrm{H}$ to $\mathrm{Xe}$ and for several series of isoelectronic molecules. The study showed that $\langle\rho\rangle$ can be actually taken as a measure to quantify electron density concentration: the more locally concentrated the electron density distribution, the larger the $\langle\rho\rangle$ value. For example, $\langle\rho\rangle_{\mathrm{Ne}}$ is larger than $\langle\rho\rangle_{\mathrm{HF}}$ because, even though they are isoelectronic systems, the same amount of electron density is more locally concentrated in the $\mathrm{Ne}$ atom than in the $\mathrm{HF}$ molecule. Consequently, $I(\mathbf{0})_{\mathrm{Ne}}$ $>I(\mathbf{0})_{\mathrm{HF}}$. Evaluation of $\langle\rho\rangle$ for the $\mathrm{C}_{2} \mathrm{H}_{2}, \mathrm{C}_{2} \mathrm{H}_{4}$, and $\mathrm{C}_{2} \mathrm{H}_{6}$ series of molecules yields 63.312, 63.388, and 63.460 a.u., respectively [which correspond exactly to four times the $I(\mathbf{0})$ values reported in Tables I and II for $\mathrm{C}_{2} \mathrm{H}_{2}$ and $\left.\mathrm{C}_{2} \mathrm{H}_{4}\right]$. This means that the electron density distribution at the $\mathrm{HF} / 6-31 \mathrm{G}^{*}$ level of theory follows the trend $\mathrm{C}_{2} \mathrm{H}_{6}>\mathrm{C}_{2} \mathrm{H}_{4}>\mathrm{C}_{2} \mathrm{H}_{2}$, from the more to the less locally concentrated. The main reason for the difference in the electron density concentration along this series of molecules is the two-hydrogen (i.e., two-electron) difference between each molecule, as $\langle\rho\rangle_{\mathrm{H}}$ is $\sim 0.04 .{ }^{19}$ This is quantitatively evidenced by the fact that $\Delta\langle\rho\rangle$ between two consecutive molecules in the series is practically constant $(\Delta\langle\rho\rangle$ $=0.072$ a.u. between $\mathrm{C}_{2} \mathrm{H}_{6}$ and $\mathrm{C}_{2} \mathrm{H}_{4}$ and $\Delta\langle\rho\rangle=0.076$ a.u. between $\mathrm{C}_{2} \mathrm{H}_{4}$ and $\mathrm{C}_{2} \mathrm{H}_{2}$ ), the final small difference between the two $\Delta\langle\rho\rangle$ values $(0.004$ a.u.) being attributed to the particular internuclear electronic reorganization in each molecule, essentially related to the strengthening of the carboncarbon bond.

An opposite behavior to the above mentioned general trend is found when comparing, on one hand, $I(\mathbf{r})$ values on the positions of the local minima in $\nabla^{2} I(\mathbf{r})$ assigned to the set of $\left\{\mathrm{C}_{i} \mathrm{C}_{j}\right\}$ interactions and, on the other hand, $E(\mathbf{R})$ values on the positions of the local minima in $\nabla^{2} E(\mathbf{R})$ assigned to the set of $\left\{\mathrm{C}_{i i}\right\}$ interactions in $\mathrm{C}_{2} \mathrm{H}_{2}$ and $\mathrm{C}_{2} \mathrm{H}_{4}$. A good explanation to these results emerges if one takes under consideration the qualitative spatial assignments stated above for the different types of electron-electron interactions. On one side, the fact that $I(\mathbf{r})$ for $\left\{\mathrm{C}_{i} \mathrm{C}_{j}\right\}$ is 16.354 a.u. in $\mathrm{C}_{2} \mathrm{H}_{2}$ and 15.869 a.u. in $\mathrm{C}_{2} \mathrm{H}_{4}$ is mainly due to the contribution of the additional set of $\left\{\mathrm{C}_{i} \mathrm{H}_{i}\right\}$ interactions to the same spatial region in $\mathrm{C}_{2} \mathrm{H}_{2}$. On the other side, the fact that $E(\mathbf{R})$ for $\left\{\mathrm{C}_{i i}\right\}$ is 70.848 a.u. in $\mathrm{C}_{2} \mathrm{H}_{2}$ and 66.931 a.u. in $\mathrm{C}_{2} \mathrm{H}_{4}$ can be mostly attributed to the contribution of the additional set of $\left\{\mathrm{C}_{i} \mathrm{H}_{j}\right\}$ interactions to the same spatial region in $\mathrm{C}_{2} \mathrm{H}_{2}$.

Among the different electron-electron interactions mentioned above, the assignment to a local minimum in the $\nabla^{2} E(\mathbf{R})$ maps of what have been identified as $\left\{\mathrm{H}_{i i}\right\}$ interactions deserves some additional comments. Their presence can be better understood if one considers this kind of electron-electron interactions as a reflect of, using a valencebond language, the contribution of ionic structures (formally represented as $\mathrm{H}^{-}$) to the total molecular wave function. As it is well known, under the Hartree-Fock approximation the contribution of ionic structures is strongly exaggerated, and this fact could explain their clear identification in Figs. 1(d) and 2(d). The use of wave functions accounting for electron correlation effects should diminish the extent of the region assigned to $\left\{\mathrm{H}_{i i}\right\}$ interactions where $\nabla^{2} E(\mathbf{R})$ is locally concentrated or even eliminate their appearance in the present $\nabla^{2} E(\mathbf{R})$ maps. $^{16}$

Up to this point, no specific reference to electronelectron interactions involving electrons of the carbon valence shell has been made to simplify the arguments leading to a fundamental understanding of the interpretative aspects of $\nabla^{2} I(\mathbf{R})$ and $\nabla^{2} E(\mathbf{R})$ maps. This type of interactions certainly contribute to some extent to the different local minima regions, and thus at this stage it would be interesting to discuss the possibility of its identification in Laplacian maps. For this purpose, calculations of $I(\mathbf{r})$ and $\nabla^{2} I(\mathbf{r})$ for $\mathrm{C}_{2} \mathrm{H}_{2}$, $\mathrm{C}_{2} \mathrm{H}_{4}$, and $\mathrm{C}_{2} \mathrm{H}_{6}$ along the $x$ axis have been performed, and the results are depicted in Fig. 3. At first look, the shape of $\nabla^{2} I(\mathbf{r})$ for $\mathrm{C}_{2} \mathrm{H}_{2}$ [Fig. 3(a)] reveals two new local minima that were not previously considered in Table I. The position on the $x$ axis (marked with a dotted line) of the first new local minimum is found at 1.022 a.u. (between the local minima assigned to the 0 and $\left\{C_{i} C_{j}\right\}$ interactions in Table $\mathrm{I}$ ), while the second is located at 3.343 a.u. (between the local minima assigned to the $\left\{\mathrm{C}_{i} \mathrm{C}_{j}\right\}$ and $\left\{\mathrm{C}_{i} \mathrm{H}_{j}\right\}$ interactions in Table I).

Focusing our attention in the first new local minimum $(x=1.022$ a.u. $)$, it can be observed that it appears as an assymmetric well in Fig. 3(a). However, interestingly enough, inspection of the $\nabla^{2} I(\mathbf{r})$ shape for $\mathrm{C}_{2} \mathrm{H}_{4}$ [Fig. 3(b)] evidences a shoulder in the spatial position where the single asymmetric well was originally located, and it envolves towards a double well in the shape of $\nabla^{2} I(\mathbf{r})$ for $\mathrm{C}_{2} \mathrm{H}_{6}$ [Fig. $3(\mathrm{c})]$. In this latter case, the two local minima of the double well are located at 1.015 and 1.803 a.u., thus showing that the position of the local minimum in the original assymmetric well has been approximately maintained. It seems then clear that the original single local minimum and the final two local minima can be assigned to electron-electron interactions involving electrons of the carbon valence shell; in $\mathrm{C}_{2} \mathrm{H}_{2}$ the valence shells of the two carbons almost overlap completely, and the sets of core electron-valence electron intrac- 

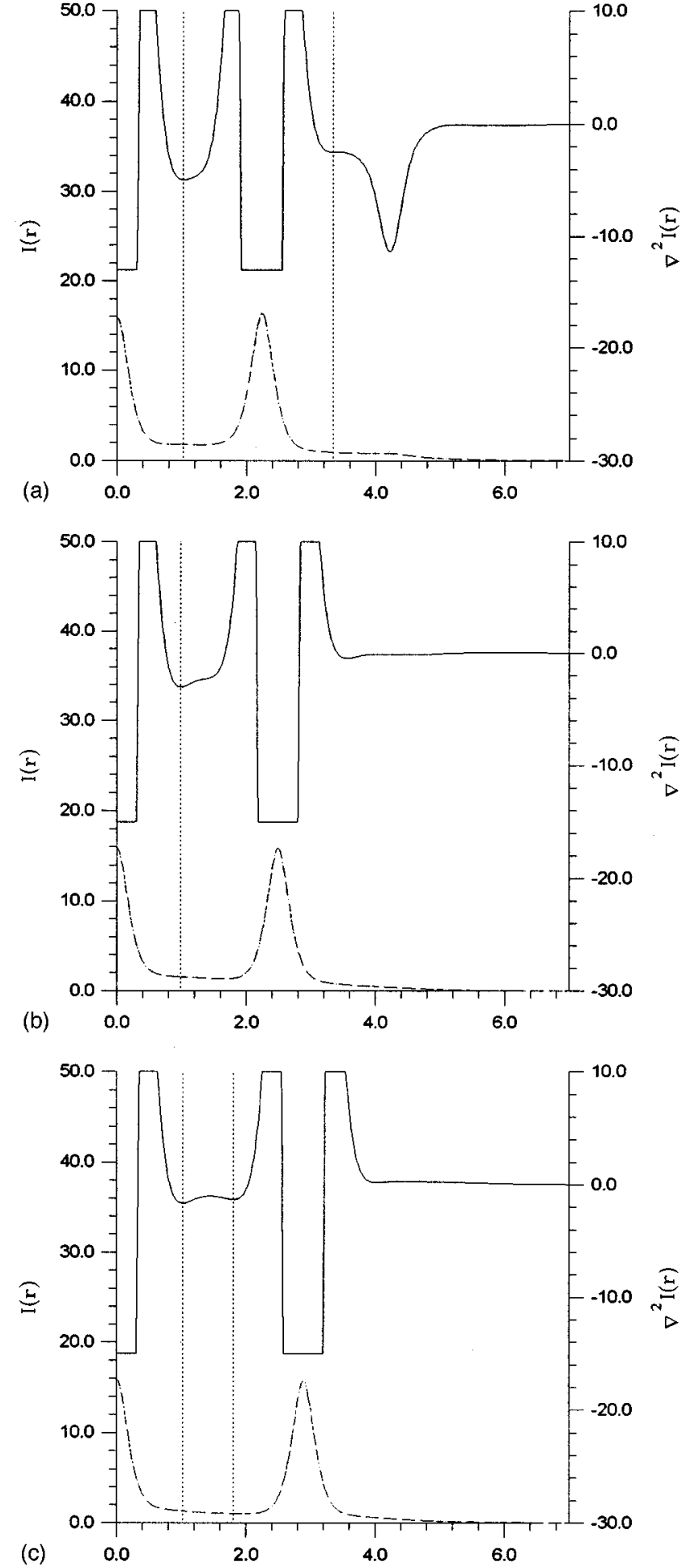

FIG. 3. Intracule density (dashed lines) and its Laplacian (solid lines) for (a, top) $\mathrm{C}_{2} \mathrm{H}_{2}$, (b, middle) $\mathrm{C}_{2} \mathrm{H}_{4}$, and (c, bottom) $\mathrm{C}_{2} \mathrm{H}_{6}$ along the axis defined by the two carbon atoms.

arbon $\left(\left\{\mathrm{C}_{i} \mathrm{C}_{i}\right\}\right)$ and intercarbon $\left(\left\{\mathrm{C}_{i} \mathrm{C}_{j}\right\}\right)$ interactions furnish a single local minimum; as the carbon-carbon distance is lengthened, the $\left\{\mathrm{C}_{i} \mathrm{C}_{i}\right\}$ interactions remain at about the same position, whereas the set of $\left\{\mathrm{C}_{i} \mathrm{C}_{j}\right\}$ interactions begin to appear at larger distances, appearing finally in the form of a double well for the $\mathrm{C}_{2} \mathrm{H}_{6}$ molecule. The use of this kind of analysis on a series of related molecules can be a good strategy when attempting to separate contributions from different sets of electron-electron interactions in about the same spatial region.

\section{CONCLUSIONS}

The topologies of intracule and extracule densities have been compared to those of their respective Laplacians for the $\mathrm{C}_{2} \mathrm{H}_{2}, \mathrm{C}_{2} \mathrm{H}_{4}$, and $\mathrm{C}_{2} \mathrm{H}_{6}$ series of molecules. While only two local maxima were rigorously characterized in $I(\mathbf{r})$ and $E(\mathbf{R})$ maps, identification of the local minima in the topology of $\nabla^{2} I(\mathbf{r})$ and $\nabla^{2} E(\mathbf{R})$ distributions allowed a more detailed analysis of the different types of electron-electron interactions present in the molecules and permitted the assignment of their most probable spatial situation. However, when compared to the ease of interpretation of molecular one-electron densities and Laplacians, it has been shown that a correct interpretation of molecular intracule and extracule density and Laplacian distributions requires a much more careful examination. The fact that several electron-electron interactions may contribute to close regions in space (specially in intracule distributions) introduces an additional difficulty when trying to perform a precise interpretation of the maps and attempt a quantitative study of the contribution of each particular interaction. Despite these inherent difficulties, the relevance of the Laplacian of intracule and extracule distributions for analyzing electron-electron interactions in molecules has been clearly manifested. This property makes the topology of these Laplacians a particularly promising tool to be applied in the study of a wide range of aspects in chemical problems. More research in this direction is underway in our laboratory.

\section{ACKNOWLEDGMENTS}

This work has been supported by the Spanish DGICYT Project No. PB95-0762. X.F. benefits from a Doctoral fellowship from the University of Girona. We thank also the Centre de Supercomputació de Catalunya (CESCA) for a generous allocation of computing time.

${ }^{1}$ R. F. W. Bader, Atoms in Molecules: A Quantum Theory (Clarendon, Oxford, 1990).

${ }^{2}$ J. Cioslowski and G. Liu, J. Chem. Phys. 105, 8187 (1996).

${ }^{3}$ E. R. Davidson, Reduced Density Matrices in Quantum Chemistry (Academic, New York, 1976).

${ }^{4}$ A. J. Coleman, Int. J. Quantum Chem. 18, 457 (1967).

${ }^{5}$ A. J. Thakkar and V. H. Smith, Jr., Chem. Phys. Lett. 42, 476 (1976).

${ }^{6}$ A. E. Carlsson and N. W. Ashcroft, Phys. Rev. B 25, 3474 (1982).

${ }^{7}$ A. J. Thakkar, J. Chem. Phys. 84, 6830 (1986).

${ }^{8}$ J. Cioslowski, B. Stefanov, A. Tang, and C. J. Umrigar, J. Chem. Phys. 103, 6093 (1995).

${ }^{9}$ J. Wang and V. H. Smith, Jr., Chem. Phys. Lett. 220, 331 (1994).

${ }^{10}$ C. Sarasola, L. Domínguez, M. Aguado, and J. M. Ugalde, J. Chem. Phys. 96, 6778 (1992).

${ }^{11}$ A. J. Thakkar, A. N. Tripathi, and V. H. Smith, Jr., Int. J. Quantum Chem. 26, 157 (1984).

${ }^{12}$ M. Breitenstein, H. Meyer, and A. Schweig, Chem. Phys. 124, 47 (1988).

${ }^{13}$ J. Wang and V. H. Smith, Jr., Int. J. Quantum Chem. 49, 147 (1994).

${ }^{14}$ J. Cioslowski and G. Liu, J. Chem. Phys. 105, 4151 (1996).

${ }^{15}$ Gaussian 94, M. J. Frisch, G. W. Trucks, H. B. Schlegel, P. M. W. Gill, B. G. Johnson, M. A. Robb, J. R. Cheeseman, T. Keith, G. A. Petersson, J. A. 
Montgomery, K. Raghavachari, M. A. Al-Laham, V. G. Zakrzewski, J. V. Ortiz, J. B. Foresman, C. Y. Peng, P. Y. Ayala, W. Chen, M. W. Wong, J. L. Andrés, E. S. Replogle, R. Gomperts, R. L. Martin, D. J. Fox, J. S. Binkley, D. J. Defrees, J. Baker, J. P. Stewart, M. Head-Gordon, C. Gonzalez, and J. A. Pople, Gaussian, Inc., Pittsburgh, Pennsylvania, 1995.

${ }^{16}$ X. Fradera, M. Duran, and J. Mestres (submitted).

${ }^{17}$ As a strict separability between contributions from particular electron pairs is not completely possible under the present scheme, the notation used to refer to electron-electron interactions and their assignment to local minima regions are not exclusive and should be taken with some flexibility.

${ }^{18}$ J. M. Ugalde and C. Sarasola, Phys. Rev. A 49, 3081 (1994).

${ }^{19}$ M. Solà, J. Mestres, J. M. Oliva, M. Duran, and R. Carbó, Int. J. Quantum Chem. 58, 361 (1996). 